\title{
Scaling-up filariasis lymphoedema management into the Primary Health Care System in Kerala State, Southern India - an example of equity in healthcare.
}

Suma Krishnasastry ( $\square$ sumatk@gmail.com )

Filariasis Research Unit, WHO Collaborating Center for LF MMDP, Department of Internal Medicine, Govt.

T. D. Medical College Hospital, Alappuzha, India. 688 005. https://orcid.org/0000-0003-2305-3845

Charles D. Mackenzie

NTD Support Center, Task Force for Global Health, Atlanta Georgia 30030, USA.

Rajeev Sadanandan

Health Systems Transformation Platform, New Delhi, India 110070 https://orcid.org/0000-0001-73847788

Research Article

Keywords: Lymphatic filariasis, health equity, India, lymphoedema, GPELF

Posted Date: November 1st, 2021

DOI: https://doi.org/10.21203/rs.3.rs-1014961/v1

License: (9) This work is licensed under a Creative Commons Attribution 4.0 International License.

Read Full License 


\section{Abstract}

\section{Background:}

Lymphatic filariasis remains one the world's most debilitating parasitic infections and is a major contributor to poor health in many endemic countries. The provision of continuing care for all those affected by this infection and its consequences is an important component of the United Nations' Sustainable Development Goals. The World Health Organization's recommendation for achieving this goal is for countries to integration their lymphedema care into the primary health care system.

\section{Methods:}

To fulfil the WHO recommendation for providing care for all those affected with lymphoedema caused by lymphatic filariasis, selected health care providers from each of the endemic districts in Kerala participated in intensive training sessions endorsed by the State's medical administration. The six training sessions included appropriate self-care information and the development of individual plans for each participating institution to provide instruction and care for their lymphoedema patients. The learning achieved by attendees was assessed during the training sessions by pre- and post-training testing. The number of lymphoedema patients receiving care and instruction from the post-training activities of each participating institution was assessed from local records, six months after the conclusion of the training sessions.

\section{Results:}

One hundred and eighty-four medical personnel (91 doctors and 93 staff nurses) from 82 medical institutions were trained which quickly led to the establishment of active lymphoedema clinics providing the essential package of care (EPC) for lymphoedema patients at all the participating institutions. Six months after the training sessions the number of previously unidentified lymphoedema patients registered and receiving care at these clinics ranged from 296 to almost 400 per clinic, with a total of 3477 new patients receiving training in the EPC during this period.

\section{Conclusions:}

Generalist health personnel, when appropriately trained, can provide quality lymphoedema care in public health settings, and that patients, when provided services close to their home, are willing to access these services. This approach is a feasible strategy for integrating long term care for LF patients into a national health system, and is a clear example of moving towards equity in health care for the medically underserved, and thus successfully addresses a major goal of the global program to eliminate lymphatic filariasis (GPELF).

\section{Background}


Lymphatic filariasis (LF) remains one of the world's most debilitating parasitic infections and is a major contributor to poor health as the second most common global cause of physical disability. It is geographically limited to 72 economically challenged countries hampering their socio-economic progress [1]. In India, LF is the commonest cause of lymphoedema and hydrocele, where the nocturnally periodic form, Wuchereria bancrofti (transmitted by Culex quinquefasciatus), is responsible for $95 \%$ of these cases, and Brugia malayi (transmitted by Mansonia mosquitoes) for the remaining $5 \%$ of cases [2,3]. It is estimated that there are 450 million infected and affected people in India, accounting for $40 \%$ of the global human LF infections, with a further estimated 450 million population still 'at risk' of infection $[1,4]$ Around 7.44 million suffer from LF-induced lymphoedema in India and approximately 12.88 million are suffering from hydrocele.

In 1997 the World Health Assembly targeted for global elimination of infection as a public health problem [5]. This initiative, known as the Global Programme to Eliminate LF (GPELF), consists of two major pillars: (i) interrupting transmission using Mass Drug Administration (MDA) to prevent the disease in future generations, and (ii) alleviating the suffering in those who already have the disease through Morbidity Management and Disability Prevention (MMDP). To address the second of these pillars, the World Health Organisation (WHO) recommends an "essential package of care" (EPC) which includes a) treatment for acute attacks or adenolymphangitis (ADL), b) management of lymphedema, c) surgery for hydrocele, and d) treatment of any remaining LF infection with MDA drugs. It was also seen entail that these services should be available in all locations wherever patients are known to reside (i.e., $100 \%$ geographic availability or coverage) $[6,7]$.

An important aspect of providing care for a chronic disease such as LF lymphoedema that often affects individuals for the rest of their lives is the continuing availability of the care and support. To achieve this, in addition to the four WHO recommendations for MMDP described above, there is the important additional recommendation for the integration of this medical support into the public health system of an endemic country. Such a goal is also in line with the global goals set out by the United Nations' Sustainable Develop Goals (SDG) [8]. This integration has often been a challenge for many LF endemic countries but has been an important goal for the filariasis program in highly endemic Kerala State, Southern India.

The major aim in the integration efforts in Kerala was to ensure the availability of the WHO recommended EPC for LF patients in all areas of Kerala, South India, with the aim of providing $100 \%$ geographical coverage of the EPC for LF patients, and that at least one health facility designated for MMDP services per GPELF Implementation Unit (IU). In addition, as advised by WHO, these services should be provided at the appropriate level of the government health system, and be of good quality. This current communication describes the activities carried to fulfill this integration efforts and the successes achieved in addressing this important health initiative in this filariasis endemic region of India.

\section{Methods}




\section{Study population}

Kerala State in south western India has a population of approximately 34.7 million people and $11 / 14$ districts are know to be endemic for lymphatic filariasis (all districts except Pathanamthitta, Idukki \& Wyanad). Although GPELF was officially launched by WHO in 2000, MDA was first started in Alappuzha, Kozhikkode and Kannur districts in 1997. Subsequently the MDA activities were extended to all 11 endemic districts with diethylcarbamazine and albendazole being given in a total of eight annual rounds of MDA, the last being carried out in 2012. Currently MDA is continuing only in certain areas of one district (Malappuram) where the infection has persisted.

\section{Goals}

The Kerala LF Elimination program is the part of SDG activities of the State, with the specific SDG targets for LF established for Kerala State being:

1. Interruption of transmission by reducing microfilarial (Mf) prevalence below $1 \%$ in all endemic districts.

2. Ensuring availability of the "recommended essential package of care (EPC) " in all areas with known patients.

SDG 3.3 refers to a reduction in number of persons requiring NTD interventions, with SDG 3.8 / Universal Health Coverage (UHC) concerning access to quality essential health care services and access to safe, effective, quality and affordable essential medicines and vaccines for all. In case of LF the recommended essential package of care (lymphedema management, ADL treatment and hydrocelectomy) should be provided under the framework of Universal Health Coverage through the primary health care system with the aim of "leaving no one behind" [8]. To attain this, the LF MMDP services should be included in the essential package of services of UHC.

The fundamental strategy ultimately followed in Kerala, in keeping with WHO recommendations, involves the following major steps:

1. Knowing the patient burden: An estimate of the number of lymphedema and hydrocele cases is important for planning, and for determining the number and location of LF care-providing facilities to be established, the allocation of funding, and the subsequent assessment of success of these actions, including the overall integration of long-term LF patient care into the public health system.

2. Making MMDP services available: The aim is to have $100 \%$ geographic coverage of MMDP where each IU has at least one facility providing the EPC for lymphoedema patients and there is availability of surgical treatment for all those suffering from hydrocoele.

3. Ensuring the quality of these services: The provided services for LF patients should be of good quality; an assessment of quality should be carried out in at least $10 \%$ of the designated medical facilities using 
the WHO Direct Inspection protocol.

\section{Training procedures}

The Director of Health Services for Kerala State selected specific health facilities in every district (i.e. 14 districts), which included the three non-endemic districts as patients with LF clinical disease have been reported to reside in these districts; doctors and nurses from these health facilities were then selected for the training. The training on MMDP was inaugurated by the Additional Chief Secretary Shri. Rajeev Sadanandan on $5^{\text {th }}$ June 2017 at Govt. TD Medical College, Alappuzha. The training was given to a total of 184 health care providers, in 6 sessions of 3 days each, by the staff of the Filariasis Research Unit, Govt. TD Medical College, Alappuzha. The number of these health care providers that were trained and the dates of the training events are given in Table 1.

The training content followed the WHO Certified Training Module which encompasses components detailing the general background, as well as clinical and programmatic, aspects of LF. The learning objectives of the training sessions for the attendees were to: 1) to understand the background, the requirements and current status of global program to eliminate LF (GPELF); 2) to understand LF clinical disease focusing on lymphoedema, hydrocele, and acute attacks (ADL); also to be aware of the recommended essential package of care; and to 3 ) learn details concerning the implementation of GPELF including situation analysis, selection and development of health facility to impart MMDP services, the documentation and reporting of services provided, and assessment of quality of services given by the health facility.

The longstanding WHO Filariasis Center in the Government TD Medical College of Alappuzha [6, 8], worked with Kerala's medical Administration to develop a solid, state wide, system of specialised care for LF lymphoedema patients. The training of the medical staff occurred through organized, structured sessions over six months included information, hands-on demonstrations and the development of specific LF lymphoedema care plans for the trainees' hospitals (Figure 1).

\section{Assessment of activities and outcome}

Each training session included interactive sessions, photo quizzes, as well as direct interaction with lymphoedema patients. Participants were instructed in taking clinical history, carrying out a physical examination, as well aa being given hands-on-training on limb hygiene and other details of lymphedema management. The participants were divided into groups according to their institutions and each group given the task of developing a proposal for initiating MMDP services at their own hospitals. The institutional plans that each group of participants developed during the training sessions were assessed for quality and content. At the beginning of each of the six training sessions every attendee carried out a twenty item questionnaire with questions covering both programmatic and clinical elements. The same questions were asked again at the conclusion of each training session. A few attendees (an overall total of 8 across all sessions) did not carry out both tests. Throughout the training period regular 
conversations were held with the participants to obtain their opinions of the training and on any challenges they might have had in implementing the instructions and advice they received.

The number of medical facilities that instigated a lymphoedema care system as result of attending the training sessions was assessed one year after the training sessions ended. The number of patients who received instruction in the package of self-care from these clinics at 6 months after the trading session was also determined by survey of all participating institutions.

\section{Results}

\section{Training sessions}

One hundred and eighty-four medical personnel (91 doctors and 93 staff nurses) from 82 medical institutions were trained which led to the immediate establishment of lymphoedema clinics at all these institutions to provide the EPC for these patients (Table 1). Interviews with all the participants in all the six different sessions found that they were very enthusiastic about the training sessions and the included activities; participants were especially satisfied with the development, as part of the training program, of quality institutional proposals for starting and carrying out MMDP services for their own patients suffering from LF. These proposals included appraising their Superintendents and DMOs about the need for initiating the MMDP services, the training of the other health care providers in their own hospitals, carrying out infrastructure modifications, procuring the necessary materials for management of lymphedema and acute attacks, i.e., antibiotics and other drugs, antiseptic, anti-fungal ointments, and the acquisition of the necessary materials for limb hygiene measures. These plans also included introduction of appropriate IEC activities and maintenance of records and documents, as well planning for regular documentation and reporting on their ongoing activities.

An important factor in the success of these training sessions was the support from the State Administration. This was seen firstly in the instructions put forward by the Additional Chief Secretary who recommended that to sustain the essential capability and knowledge for LF patient care to be provided in the upcoming training sessions, a team of health care providers - a doctor and staff nurse - from each Taluk Head Quarters hospitals should be given training in LF MMDP. His vision was that these teams could then train others in their respective facilities, and thus ensure a wide distribution of the needed services across the State. The second example of important administrative support for the training activities was the attendance of high-level government officials at the opening of each session: this underscored the importance of these training sessions.

A comparison of the pre-training tests with those taken after the training session was completed showed a very high degree of learning achieved by virtually all attendees. Figure 3 shows the pre-training and post-training scores obtained by the attendees at each session who took both the pre- and post-training tests. Before training the number of questions answered correctly across the six sessions ranged from an average of 9.4 (with a range between groups of 8.6 to 10.6 of the 20 questions answered correctly; after 
training this improved to $16.7(16.1$ - 20.0). Thus, across all the training sessions there was almost twice as many questions being answered correctly after training, and in each group there were attendees who got all questions correct or only answered one or two questions incorrectly. The institutional planning projects were all examined and found to be of high quality and to contain all the major needed components for implementation.

\section{Post training activities}

\section{MMDP Clinics}

On returning to their posts after the training session, the participants had discussions with their Superintendents and DMOs, organized training program for doctors, nurses and health care workers and carried out various IEC activities. Importantly the State Government allocated funds for the LF MMDP activities in every district as part of the SDG program and now all the districts with clinical LF have started MMDP clinics. Twelve months after the training sessions were completed a total of 82 MMDP clinics had been started in the State as part of the overall SDG program with an average of 5 facilities per district.

\section{Increases in patients receiving care}

The training of the medical staff occurred through organised structured sessions over six months that included information, hands-on demonstrations and the development of specific LF lymphoedema care plans for the trainees' home hospitals and medical centers (Figure 1).

In the following six months after the training the number of previously unidentified lymphoedema patients registered and receiving care at these clinics ranged from 296 to almost 400 per clinic, with a total of 3477 new patients receiving the EPC during this period.

\section{Discussion}

Lymphatic filariasis affects mainly socioeconomically poor people and the infection usually occurs early in life, while the clinical disease appears later in life. The chronic manifestations of the disease are lymphoedema (LE), " elephantiasis" and hydrocœle [9]. LF causes lymph vessel dilatation and lymphatic dysfunction, and lymphatic changes occur even in asymptomatic individuals especially children; this lymphatic dysfunction predisposes the affected areas to recurrent bacterial infections. Consequently, these secondary infections precipitate acute adenolymphangitis (ADL) acute or clinical attacks, the commonest acute manifestation of LF. The bacteria commonly gain access to the parasite-damaged lymphatic vessels through dermal injury, skin compromised by chronic fungal infections, fissures, and paronychia - all commonly called entry lesions. ADL episodes usually worsen the overall lymphoedema, and as the condition develops there is a resulting increase in frequency of the ADL attacks resulting in a self-promoting vicious cycle. The more advanced form of lymphoedema usually presents with marked dermal changes, and is commonly known as "elephantiasis". Hydrocele, the other major manifestation of 
lymphatic filariasis, results from the accumulation of fluid in the cavity of the tunica vaginalis of the scrotum; this condition can be successfully treated surgically. Patients with LF disease suffer substantial physical, social, economic, psychological and sexual disabilities.

The impairment and disability resulting from the LF-associated lymphoedema, elephantiasis and hydrocele cause a significant public health problem; however, there are relatively simple medical interventions that can address these problems and assist patients. These include treatment for acute attacks ( $A D L$ ), the reduction in the frequency and severity of $A D L$ with simple hygiene measures, such as washing and basic skin care; which assist in preventing progression of the lymphoedema to the stage of elephantiasis. The currently recommended treatment for bouts of ADL is administration of antibiotics and other supportive measures. The hygiene measures used include: washing the affected parts twice daily with soap and clean water at room temperature and drying carefully with a clean cotton cloth (especially between the toes); maintaining clean nails and treating interdigital lesions (usually with antifungal creams) ; avoiding 'entry' lesions through using proper footwear and the use of antiseptic or antibiotic creams to treat small wounds or abrasions. For management of hydrocele, surgical intervention with a hydrocelectomy is the standard option; this is usually a relatively uncomplicated surgical intervention if carried out using adequate surgical procedures and appropriate pre- and post-surgery management.

Prior to the initiation of the GPELF those affected by the lymphedema induced by this parasitic infection, and who suffer the consequent disability and compromised quality of life, have often been unable to obtain the needed care except in a few specialised clinics in concerned countries, such as India, Sri Lanka and Brazil $(3,10,11,12)$. The major requirement of GPELF to provide care for all those clinically affected is an example of progress towards restoring health equity a global goal, and in this case equity for a group of people who have, at least until recently, largely been ignored. It is vital that LF patients suffering from parasite-induced lymphoedema obtain the care as defined by GPELF so that these patients have a fair opportunity to reach their full health potential through the provision of care for their lymphoedematous condition [5]. This requires that the appropriate care they need is available through, not only through specialised clinics, but also thorough the formal public health system of an endemic area.

Arguably the two most important factors to the success of this overall venture were, firstly the engagement of the involvement of highest level of Government medical officials, and secondly providing medical staff with added medical skills and capability. These two factors have often been difficult to achieve in many endemic countries, although those that have managed to incorporate these components have often achieved success. In this current study the presence of the Additional Chief Secretary of Health at the opening session, and his instructions regarding required participation and attendance, were clearly catalytic in the success of the training sessions. It is, in general, logically better to follow WHO recommendation to carry out a situational analysis and assessment of local disease LE burden before including specific health facilities in training sessions; however, here in Kerala this was not done, and the inclusion of a particular health facility in training sessions was based on local knowledge of the presence of these patients in the area. It is important to note that the attendees of the training sessions indicated 
that they had not previously been taking adequate care of LF patients due to a lack of awareness of the required procedures and a lack of facilities. Following the training, the attendees realised that with their newfound knowledge they were now capable of providing quality care to LF patents in an easily affordable manner and that it actively improved the quality of life of their patients. The reported that direct interaction with LF patients and the hands-on activities during training gave them confidence to manage these neglected patients, to become champions for LF care, and to be able train other health workers in their medical institutions.

The activities presented in this paper, with the training of 184 medical personnel from 82 institutions, are an example of successful integration of healthcare into the public health system in an endemic area. Over three years the longstanding WHO Filariasis Center in the Government TD Medical College of Alappuzha, Kerala State, worked with Kerala State government medical officials to develop a solid state wide system of specialised care for LF lymphoedema patients, and have provided a strong example of achieving equity in healthcare. It is also a major step towards achieving the goals of GPELF for the people of Kerala. Kerala is the first State in India to achieve this integration of LE care into the public health system, and arguably one of the first amongst all global endemic countries. The approaches taken here in Kerala are likely to be useful in other countries and Indian states. Kerala's success in imparting MMDP services to LF patients throughout Kerala is being recognized by the international NTD community as the "Kerala story".

\section{Conclusions}

This Kerala approach to increasing the provision of support for lymphoedema self care has demonstrated that generalist health personnel, when appropriately trained, can provide quality lymphoedema care in public health settings, and that patients, when provided services close to their home, are willing to access these services. The key factors in achieving this success in Kerala, which are likely applicable to many situations across the LF endemic world, were:

1. Engaging the support and involvement of senior Government officials from the Health and Family Welfare Department.

2. Ensuring the engagement of medical staff from all endemic districts.

3. Participatory training sessions with both active practical and planning components.

This approach, a feasible strategy for integrating long term care for LF patients into a national health system, is a clear example of moving towards equity in health care for the medically underserved, and successfully addresses an essential target of the global program to eliminate lymphatic filariasis (GPELF). 


\section{Declarations}

\section{Acknowledgements}

We would like to thank all the different team members involved in this initiative, especially the government officials, the clinical officers, the nurses, and the patients.

\section{Authors' contributions}

SK carried out the planning and implementation of the overall activities, wrote the original draft of the manuscript, and co-authored the final manuscript. CM analysed data, formulated and co-authored the final manuscript. RS contributed to the design and implementation of the training programme, and contributed to the final manuscript from health policy maker's perspective.

\section{Funding}

All the Kerala based activities described in this manuscript (planning, advocacy, training and implementation) were supported by the Government of Kerala through the Department of Health and Family Welfare, and by the Govt. T. D. Medical College, Alappuzha.

In addition, the publication of the completed study was supported by the Coalition for Operational Research on Neglected Tropical Diseases (COR-NTD), which is funded at The Task Force for Global Health primarily by the Bill \& Melinda Gates Foundation (OPP1190754), by UK aid from the British government, and by the United States Agency for International Development through its Neglected Tropical Diseases Program. Under the grant conditions of the Foundation, a Creative Commons Attribution 4.0 Generic License has already been assigned to the Author Accepted Manuscript version that might arise from this submission.

\section{Availability of data and materials}

All data included in this manuscript is available from the primary institute.

\section{Declarations}

The authors have no conflict of interest.

\section{Competing interest}

The authors have no competing interests

\section{Ethics approval and consent to participate}

Ethics approval is not required for medical trading activities and their assessment.

\section{Consent for publication}


Not applicable

The participants of the MMDP training have consented to participate and/or publish and to have their image within the manuscript

\section{Abbreviations}

LF lymphatic filariasis; LE lymphoedema; GPELF Global Program for the Elimination of Lymphatic filariasis; MMDP Morbidity Management and Disability Prevention; ECP essential care Package; DMDI Disease Management and Disability Inclusion; ADL adenolymphangitis ; NTD Neglected Tropical Diseases; SDG Sustainable Development Goals.

\section{References}

1. World Health Organization. (2015) Global programme to eliminate lymphatic filariasis: progress report 2014.Weekly Epidemiological Record No.38, 2015;90:489-504.

2. Shenoy RK, Suma TK, Rajan K, Kumaraswami V. (1998) Prevention of acute adenolymphangitis in brugian filariasis: comparison of the efficacy of ivermectin and diethylcarbamazine, each combined with local treatment of the affected limb. Annals of tropical medicine and parasitology. 1998; 92(5):587-94. PMID:

3. Suma TK, Shenoy RK, Kumaraswami V. Efficacy and sustainability of a foot-care programme in preventing acute attacks of adenolymphangitis in Brugian filariasis. Tropical Medicine and International Health, 2002, 7:763-766.

4. Cromwell EA, Schmidt CA, Kwong KT, Pigott DM, Mupfasoni D, Biswas G, et al 2020. The global distribution of lymphatic filariasis, 2000-18: a geospatial analysis. Lancet Glob Health. 2020;8:e1186-94.

5. World Health Organisation. WHA 50.29. Elimination of lymphatic filariasis as a public health problem. Hbk Res., Vol. III (3rd ed.), 1.16.3.3; 7.1.3. Ninth Plenary Meeting, 13 May, 1997.

6. Mackenzie CD, Mante S (2020) Caring for patients in the global programme to eliminate lymphatic filariasis. International Health. 13 (Supplement 1):S48-S54. DOI:10.1093/inthealth/ihaa080.

7. World Health Organisation. Lymphatic filariasis. An aide memoire for LF program managers. Second edition. WHO/HTM/NTD/PCT/2016. Web Annex A. Protocol for evaluating minimum package of care of morbidity management and disability prevention for lymphoedema management in designated health facilities. 2021 https://apps.who.int/iris/bitstream/handle/10665/339870/9789240017085-eng.pdf

8. WHO (2015). Towards a monitoring framework with targets and indicators for the health goals of the post-2015 Sustainable Development Goals. Draft, WHO, January 2015.

9. Addiss DG, Brady MA. (2007)_Morbidity management in the Global Programme to Eliminate Lymphatic Filariasis:'a review of the scientific literature. Filaria journal. 2007; 6:2. doi: 10.1186/1475- 
2883-6-2

10. Shenoy RK, Suma TK, Kumaraswami V. A qualitative study on the feasibility and benefits of foot hygiene measures practiced by patients with Brugian filariasis. Journal of Communicable Diseases, 2003, 35:9-16

11. PAHO. Brazil moves towards the elimination of the transmission of Lymphatic Filariasis. https://www3.paho.org/hq/index.php?option=com_content\&view=article\&id=9399:2014-brasilavanza-hacia-eliminacion-transmision-filariasis-linfatica\&ltemid $=40264 \&$ lang $=e n$

12. Morbidity management and disability prevention for lymphatic filariasis in Sri Lanka: Current status and future prospects. PLoS Negl Trop Dis. 2018 May; 12(5): e0006472. doi:

10.1371/journal.pntd.0006472 PMCID: PMC5963805PMID: 29746479

\section{Tables}

Table 1. Attendees at the Lymphatic Filariasis MMDP training sessions held in Kerala State India (20172018)

\begin{tabular}{|c|c|c|c|c|c|}
\hline Sessions & $\begin{array}{l}\text { Training } \\
\text { session } \\
\text { dates }\end{array}$ & $\begin{array}{l}\text { Duration of } \\
\text { training } \\
\text { session }\end{array}$ & $\begin{array}{l}\text { Number of } \\
\text { doctors } \\
\text { present }\end{array}$ & $\begin{array}{l}\text { Number for } \\
\text { Staff Nurses } \\
\text { present }\end{array}$ & $\begin{array}{l}\text { Total medical } \\
\text { personnel } \\
\text { present }\end{array}$ \\
\hline 1 & $\begin{array}{l}5-8 \text { June } \\
2017\end{array}$ & 3 & 16 & 17 & 33 \\
\hline 2 & $\begin{array}{l}21-23 \\
\text { August } \\
2017\end{array}$ & 3 & 13 & 15 & 28 \\
\hline 3 & $\begin{array}{l}29-31 \\
\text { August } \\
2017\end{array}$ & 3 & 15 & 15 & 30 \\
\hline 4 & $\begin{array}{l}\text { 7-9 March } \\
2018\end{array}$ & 3 & 15 & 15 & 30 \\
\hline 5 & $\begin{array}{l}22-24 \\
\text { March } \\
2018\end{array}$ & 3 & 17 & 17 & 34 \\
\hline 6 & $\begin{array}{l}23-25 \text { May } \\
2018\end{array}$ & 3 & 15 & 14 & 29 \\
\hline $\begin{array}{l}\text { Total } \\
\text { Participants }\end{array}$ & & & 91 & 93 & 184 \\
\hline
\end{tabular}

\section{Table 2 - The number of lymphatic filariasis (MMDP-active) clinics started in Kerala Districts (as reported mid-2018)}




\begin{tabular}{lllll} 
Number & District & $\begin{array}{l}\text { No. of } \\
\text { staff } \\
\text { trained }\end{array}$ & $\begin{array}{l}\text { No. institutions } \\
\text { receiving training }\end{array}$ & $\begin{array}{l}\text { No. institutions where } \\
\text { clinics have started }\end{array}$ \\
\hline 1 & Thiruvananthapuram & 11 & 6 & 6 \\
\hline 2 & Kollam & 14 & 7 & 7 \\
\hline 3 & Pathanamthitta & 6 & 4 & 4 \\
\hline SUMA & Alappuzha & 20 & 6 & 10 \\
\hline 4 & & & & 3 \\
\hline 5 & Kottayam & 6 & 3 & 3 \\
\hline 6 & Idukki & 6 & 3 & 7 \\
\hline 7 & Ernakulam & 14 & 7 & 3 \\
\hline 8 & Thrissur & 13 & 5 & 5 \\
\hline 9 & Palakkad & 28 & 13 & 11 \\
\hline 10 & Malappuram & 21 & 9 & 9 \\
\hline 11 & Kozhikode & 18 & 10 & 1 \\
\hline 12 & Wayanad & 2 & 1 & 6 \\
\hline 13 & Kannur & 12 & 4 & 7 \\
\hline 14 & Kasaragod & 13 & 4 & 82 \\
\hline & Total & 184 & $\mathbf{8 2}$ & 3 \\
\hline
\end{tabular}

Figures 


\section{KERALA STATE (11/14 Districts)}

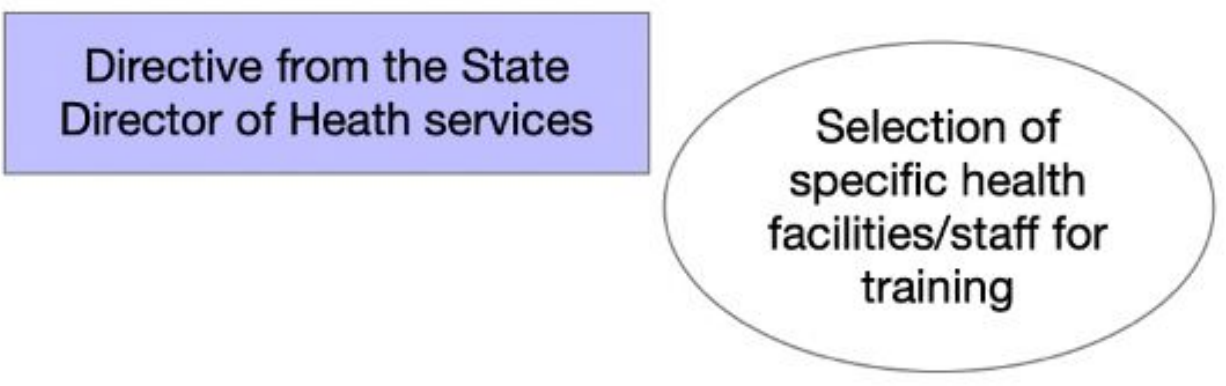

Training sessions

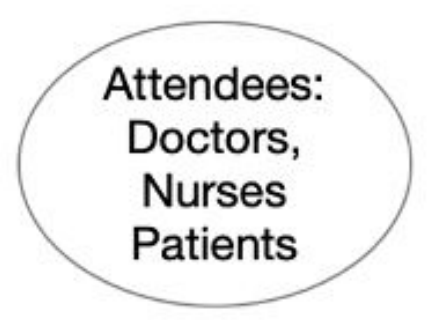

\section{6 sessions of 3 days each}

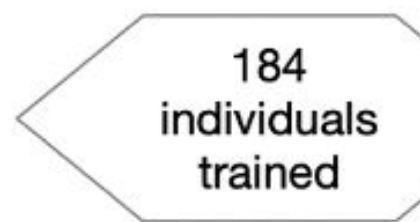

\section{Outcomes}
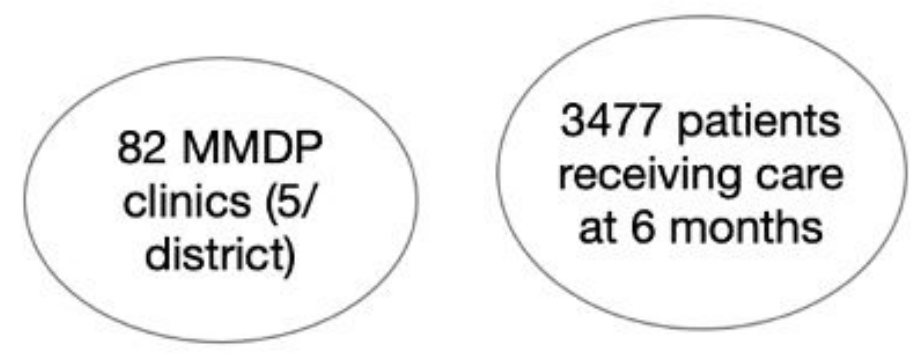

\section{Figure 1}

Flow diagram of procedures, major activities and outcomes activities in the Kerala Program.<smiles>[CH]1[CH]C1</smiles>

Figure 2 
Training of medical staff in lymphoedema care. A. Opening of training session. B. Staff developing LE care plans for their individual institutions

\section{AVERAGE BEFORE}

AVERAGE AFTER

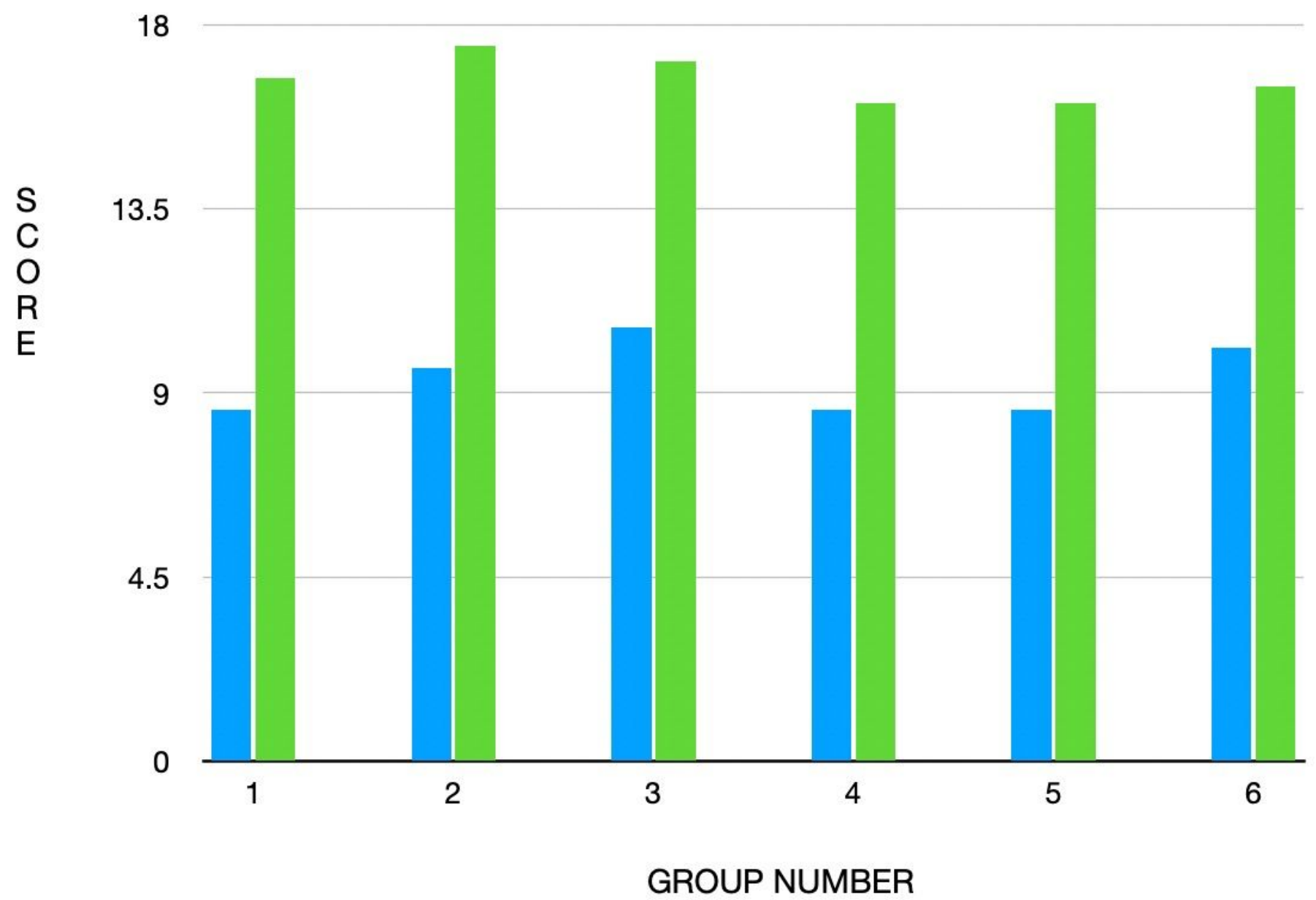

Figure 3

Comparison of the pre-test and post-test scores for 20 questions asked of each participant in each of the 6 different training session groups.

\section{Supplementary Files}

This is a list of supplementary files associated with this preprint. Click to download.

- Supplementarymaterialrevised.pdf

- Graphicalabstractrevised.jpg 